\title{
Office Endoscopy in Gynecology
}

Ambulatory or office endoscopy procedures are optimally suited to enhance effectiveness and efficiency of health care delivery systems. All the advantages of endoscopy as a diagnostic or surgical modality are maintained with a minimization of time spent in a healthcare setting. The savings in terms of time, admission, nursing and facility maintenance costs can be substantial. They could be utilized as important components of "one-stop" clinics for conditions like abnormal uterine bleeding, infertility and pain management. Office endoscopy procedures have been in practice in other specialties such gastroenterology and otorhinolaryngology since a couple of decades and are well established in these fields. In gynecology, endoscopic procedures such as colposcopy are routinely practiced as office procedures. A number of hysteroscopies (diagnostic and operative) have the potential to be converted from hospital to office settings. Endoscopy techniques such as tranvaginal hydrolaparoscopy (TVHL) and microlaparoscopy have been described for office settings. ${ }^{1}$ These procedures have become possible due to the advances in technology related to endoscopic equipment making, especially small-diameter optics, high intensity light sources and digital cameras to capture and store images.

Patient selection is paramount for successful office endoscopy. Women with obvious pathology requiring complex surgical interventions and medically unfit patients who may need supportive care are not suitable for office procedures. One of the challenges of office endoscopy procedures is to manage apprehensiveness, anxiety and pain. Counseling women and preparing them mentally are important to reduce anxiety. Premedication with oral ibuprofen reduces pain perception and oral sedation with agents such as lorazepam may be used. Atropine reduces the risk of vasovagal symptoms.
The procedure may be carried out without anesthesia, under local anesthesia or with intravenous sedation techniques depending on the procedure and comfort levels of the patient and physician. ${ }^{2}$

Hysteroscopy in the office can be performed using carbon dioxide or saline as distension medium. Carbon dioxide has the advantage of being non-messy. A special hysteroflator is needed for this. The insuffulator used for laparoscopies cannot be used due to the very large pressures it generates. Most gynecologists practicing office hysteroscopy perform it using the standard technique as used under general anesthesia. The procedure is likely to be painful and not tolerated well in these situations. This is especially true when the patient is nulliparous or menopausal. Some modifications suggested to the standard technique are the use of a $3.5 \mathrm{~mm}$ hysteroscope (as compared to a $5 \mathrm{~mm}$ sheath), local anesthesia (paracervical block), avoiding holding the anterior lip of the cervix and avoiding dilatation of the cervix. ${ }^{1}$ Bettocchi et al have pioneered the vaginoscopic approach to hysteroscopy. No instruments are introduced into the vagina except for the hysteroscope. A special Bettocchi hysteroscopy system has been designed to allow complex procedures to be performed in office settings. A recent review describes the successful application of office hysteroscopy in a wide variety of hysteroscopic procedures including biopsy, polypectomy, sterilization, metroplasty and myomectomy. ${ }^{3}$ It should be borne in mind that this group has a vast operative experience. The generally encountered problems include vasovagal episodes $(<1 \%)$ and inability to complete the procedure due to technical difficulties or pain $(\sim 5 \%){ }^{1}$

Transvaginal hydrolaparoscopy is using a culdoscopic technique was described by Grodts et al. ${ }^{4}$ The Veress 
needle-cannula system was inserted in the midline approximately $15 \mathrm{~mm}$ below the insertion of the vagina wall on the cervix. Progressively, the dilator and cannula were inserted transvaginally into the pouch of Douglas, after which the dilator and Veress needle were removed and replaced by a $2.7 \mathrm{~mm}, 30^{\circ}$ rigid, wide-angled optical system that was placed in a $3.5 \mathrm{~mm}$ shaft system ensuring irrigation with saline during the procedure. This can be followed by chromopertubation. In a series of more than 150 women, there were no failed procedures or procedures interrupted due to pain. Visualization of both adnexae was confirmed in over $90 \%$ of women. Standard laparoscopy is recommended when there are abnormal findings requiring operative intervention. It is estimated that about $75 \%$ of women would avoid a standard laparoscopy if this approach is used. ${ }^{1}$

Microlaparoscopy under local anesthesia is done using a standard Verres needle insertion intraumbilically. The sheath of the Verres needle is left in place and a $2.7 \mathrm{~mm}$ rigid laparoscope is introduced through it. Carbon dioxide is insuffulated under vision to the quantity required to visualize the structures of interest. Generally $800 \mathrm{ml}$ of gas is sufficient. The relatively smaller quantity of gas minimizes patient discomfort. Ancillary ports of $2 \mathrm{~mm}$ diameter and correspondingly small hand instruments can be used for minor operative interventions. The accuracy of narrow diameter laparoscopy has been verified in a number of studies. ${ }^{5}$ The procedure is similar to standard laparoscopy in terms of risks of injuries and gas related complications but port sites are smaller and pain is significantly lesser.

Sophisticated equipment and newer techniques have now brought into reality a variety of procedures which are even less invasive than standard endoscopy. Needless to say, equipment costs, restructuring of infrastructure in the office setting, appropriate staffing, facilities to deal with emergencies and surprises are mandatory before initiating these services. Surgeons should familiarize themselves with these modified techniques to increase the repertoire of procedures that they can offer.

\section{References}

1. Marana R, Marana E, Catalano GF. Current practical applications of office endoscopy. Curr Opin Obstet Gynecol 2001;13:383-387.

2. Society of American Gastrointestinal and Endoscopic Surgeons. SAGES Guidelines for office endoscopic services. Los Angeles, November 2008. Available at http://www.sages.org/publication/id/09. Accessed on: 28 November 2010.

3. Di Spiezio Sardo A, Bettocchi S, Spinelli M, et al. Review of new office-based hysteroscopic procedures 2003-2009. J Minim Invasive Gynecol. $2010 ; 17: 436-48$.

4. Gordts S, Campo R, Brosens I. Office transvaginal hydrolaparoscopy for early diagnosis of pelvic endometriosis and adhesions. J Am Assoc Gynecol Laparosc 2000; 7:45-49.

5. Faber BM, Coddington CC. Micro-laparoscopy: a comparative study of diagnostic accuracy. Fertil Steril 1997; 67:952-954.

Dastur Adi E

MD FICOG FCPS DGO DFP FICMU FICMCH ATMF (USA)

Honorary Professor Emeritus and Dean

Sheth G S Medical College \& Nowrosjee Wadia Mat.

Hospital, Mumbai, India. 